\title{
A Generative Model for Fingerprint Minutiae
}

\author{
Qijun Zhao, Yi Zhang \\ Sichuan University \\ \{qjzhao, yi.zhang\}@scu.edu.cn
}

\author{
Anil K. Jain \\ Michigan State University \\ jain@cse.msu.edu
}

\author{
Nicholas G. Paulter Jr., Melissa Taylor \\ National Inst. of Standards and Technology \\ \{pauter, melissa.taylor\}@nist.gov
}

\begin{abstract}
Fingerprint minutiae are the most important features used by latent fingerprint examiners, as well as in automated fingerprint recognition systems. Hence, understanding the statistical distribution of minutiae is essential in many fingerprint recognition related problems, such as fingerprint individuality and fingerprint synthesis. Prior work considers the occurrence of a minutia as a random event, and mostly assumes that individual minutiae are independent of each other. Some studies also considered the clustering tendency of minutiae and the minutiae neighborhood structures. Yet, it remains unclear whether the ridge orientation field has an impact on the minutiae occurrence. This paper investigates the correlation between ridge orientation field and minutiae. Assuming that minutiae are distributed conditionally on the variation in local ridge orientation, a new generative model is proposed for fingerprint minutiae. To evaluate the proposed model, we train the model using fingerprint images in the NIST SD14 database, and simulate the minutiae in the fingerprints in the NIST SD4 database with the trained model. The experimental results show that by exploiting both the local ridge orientation variation and the neighborhood minutiae structure, the proposed model can better simulate the minutiae extracted from fingerprints than other models available in the literature.
\end{abstract}

\section{Introduction}

Fingerprints are widely used for personal identification based on their distinctive features. These features can be roughly divided into three levels. Level-1 features refer to the global characteristics of fingerprints, such as fingerprint class, singular points, and ridge orientation field. Level2 features are defined as individual ridge events, such as minutiae; two major types of minutiae are ridge endings and bifurcations. Level-3 features are fine details in ridges, such as pores and ridge contours. Among these features, minutiae are routinely utilized by fingerprint examiners and automated fingerprint recognition systems because they are

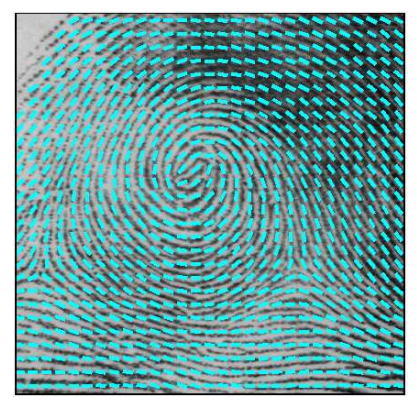

(a)

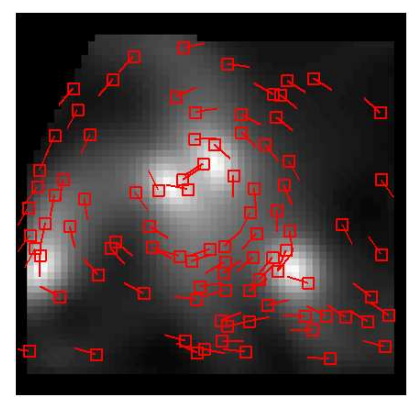

(b)
Figure 1. Correlation between minutiae occurrence and the ridge orientation field. (a) a whorl fingerprint and its ridge orientation field. (b) minutiae in (a) overlaid on the local ridge orientation deviation map. The local ridge orientation deviation (refer to Eq. (1)) indicates the variation or smoothness of the ridge orientation field in a local region. The greater the deviation of the local ridge orientation, the lower will be its smoothness.

not only discriminative but also very stable [10].

Understanding the characteristics of fingerprint features is a fundamental issue in the design, development, and deployment of fingerprint-based personal identification techniques. It is also very useful for many other problems related to fingerprint recognition, such as assessment of fingerprint individuality [11], evaluation of fingerprint recognition systems [5, 22], fingerprint feature extraction [20], indexing and matching [19], and so forth. Accurate fingerprint feature models thus are highly needed in addressing these problems.

During the past two decades, a number of models have been proposed for various fingerprint features, including singular points [6], ridge orientation field [23, 20, 19, 22], minutiae [11, 24, 7], and pores [13]. In this paper, our focus is on the statistical models for minutiae, particularly, ridge endings and bifurcations. While a number of minutiae models have been published in the literature, they do not consider the possible correlation between minutiae and ridge orientation field (see Fig. 1). Our goal in this paper is twofold: (i) to investigate the correlation between minutiae and ridge orientation field, and (ii) to incorporate 


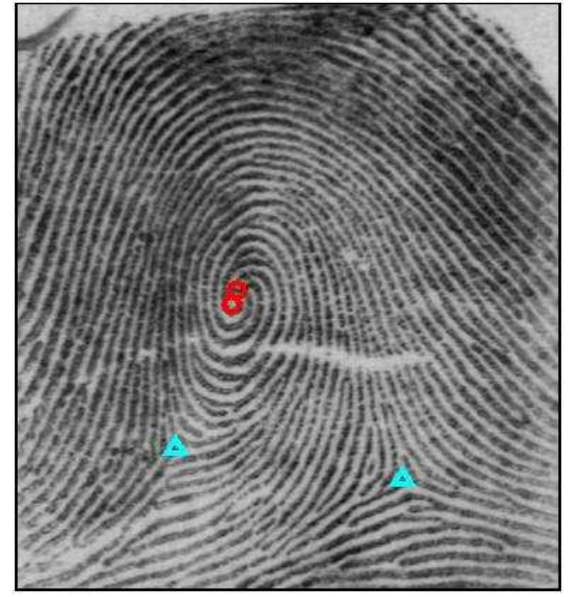

(a)

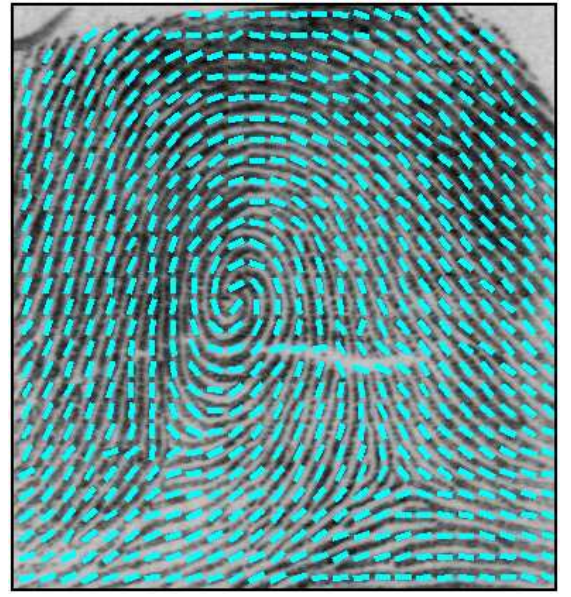

(b)

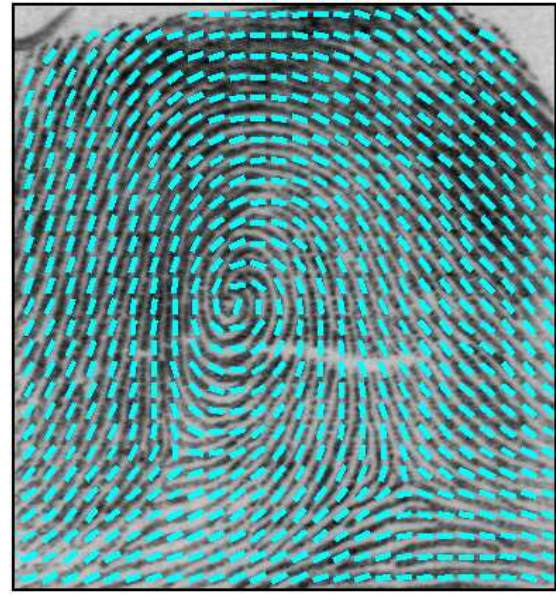

(c)

Figure 2. Estimation of fingerprint ridge orientation field. (a) a fingerprint image and its delta and core points. (b) and (c) initial and regularized ridge orientation fields of image in (a), respectively.

such correlation into the generative model of minutiae so that the model can better synthesize minutiae extracted from fingerprints.

The rest of this paper is organized as follows. In Section 2 , we review some typical minutiae models proposed in the literature. In Section 3, we study the correlation between minutiae occurrence and the ridge orientation field using fingerprints in the NIST SD14 database. Section 4 introduces the proposed generative model for fingerprint minutiae, followed by the experimental results in Section 5. Finally, Section 6 concludes the paper.

\section{Background}

Constructing statistical models of fingerprint minutiae has been a topic of significant interest in fingerprint research community. Some of the early studies on minutiae models focused on the problem of fingerprint individuality [15]. The models were used to compute the probability of random correspondence between fingerprints. However, these models could not serve as generative models for minutiae. Over the past ten years, several generative minutiae models have been proposed. Pankanti et al. [11] devised a minutiae model which assumes that (i) the occurrence of a minutia is a random event, (ii) individual minutiae are independent of each other, and (iii) the minutiae in a fingerprint are uniformly distributed. Zhu et al. [24] showed that minutiae, instead of being uniformly distributed, tend to cluster in the regions where ridge orientation changes abruptly (e.g., around delta and core points). Based on this observation, they proposed to first partition the minutiae into several clusters and then model the minutiae distributions in each cluster by using a mixture of Gaussians. The drawback of this model is that it is finger-specific; in other words, a mixture of Gaussians is learned for each finger, which makes it tedious and computationally expensive. Chen et al. [7] further improved the model of [24] by deriving a mixture of Gaussians for the fingerprints in each of the five major fingerprint classes (i.e., arch, tented arch, left loop, right loop and whorl).

Neighborhood minutiae structures were investigated by Hsu and Martin [9]. Given a central minutia, a reference local polar coordinate system is established, whose origin is at the central minutia and whose polar axis points along the direction of the central minutia. The neighborhood minutiae are represented by their positions with respect to the reference coordinate system, and the differences between their directions and the direction of the central minutia. With this representation of neighborhood minutiae, the distributions of the $k$-nearest minutiae are summarized as histograms or probability maps.

These available minutiae models either assume independence between individual minutiae or consider only the relationship among neighboring minutiae. However, they do not consider the possible correlation between minutiae and other fingerprint features. Consequently, it remains unclear whether the occurrence of minutiae (as level-2 features) are affected by level-1 features, in particular, ridge orientation field (see Fig. 1). In the next section, we will investigate the correlation between minutiae and ridge orientation field.

\section{Statistical Analysis of Minutiae Occurrence}

\subsection{Database and Feature Extraction}

To analyze the minutiae occurrence, we chose a subset of the first session fingerprint images in the NIST SD14 


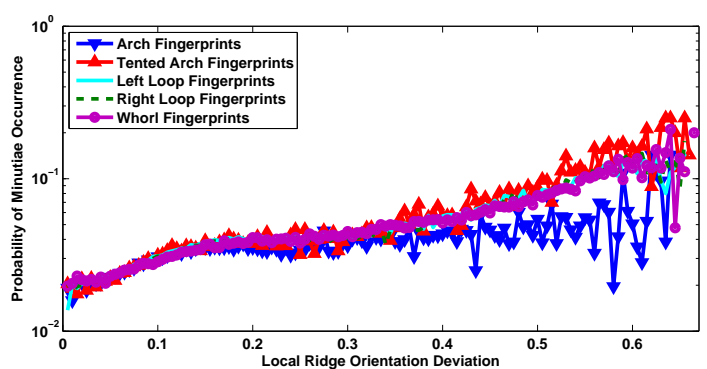

Figure 3. Occurrence probability map of individual minutiae with respect to the local ridge orientation deviation computed from the fingerprints in NIST SD14 [1].

database [1] whose NFIQ [17] values are between 1 and 3 $(\mathrm{NFIQ}=1$ means the best quality fingerprint and NFIQ $=5$ the worst quality fingerprint) as the training database. This procedure resulted in a training set consisting of 866 arch fingerprints, 302 tented arch fingerprints, 1,992 left loop fingerprints, 2,066 right loop fingerprints, and 1,434 whorl fingerprints. The foreground fingerprint region, the fingerprint type or class, and the cores and deltas in these images were manually marked by us. The minutiae were extracted by using a commercial off the shelf (COTS) fingerprint matcher, VeriFinger SDK 6.5 [2] ${ }^{1}$; minutiae outside the foreground regions were discarded. The ridge orientation field was initially estimated by using the gradient based method in [8], and then regularized by using the method described below.

Let the initial ridge orientation field be denoted as $\Theta$. As in [21], $\Theta$ is divided into singular, $\Theta_{s}$, and continuous (or residual), $\Theta_{r}$, components: $\Theta=\Theta_{s}+\Theta_{r}$. These two components are regularized separately. The singular component is determined by the singular points (if any) according to the Zero-Pole model [14], while the residual component is regularized by using the $2 \mathrm{D}$ Fourier expansion based model (FOMFE) [20]. If there are no singular points, the initial ridge orientation field is directly approximated by using the FOMFE model. The regularized ridge orientation field is able to remove most of the noise in the fingerprint (see Fig. 2). Note that the ridge orientation field is computed block-wise and the block size is $8 \times 8$ pixels $^{2}$.

To characterize the ridge orientation in a local region $\Omega_{l}$,

\footnotetext{
${ }^{1}$ Mention of any product or company does not imply endorsement or recommendation of this product or company by the authors, Sichuan University, Michigan State University, or the National Institute of Standards and Technology.

${ }^{2}$ Note that the fingerprint images used in this paper are at $500 \mathrm{ppi}$ (pixels per inch) resolution. If fingerprint images at different resolutions are used, the block size as well as the neighborhood size should be tuned accordingly.
}

we compute its deviation, $d_{\theta}$, in the local region by

$$
d_{\theta}=\frac{1}{\left|\Omega_{l}\right|} \sum_{(x, y) \in \Omega_{l}} \sin |\theta(x, y)-\bar{\theta}|,
$$

where $\left|\Omega_{l}\right|$ is the area of the local region, and $\bar{\theta}$ is the average ridge orientation in $\Omega_{l}$. In this paper, the local ridge orientation deviation at a block is computed based on its $9 \times 9$ neighboring blocks. The local ridge orientation deviation ranges from 0 to 1 with higher values denoting larger variations (or lower smoothness). See Fig. 1.

The ridge orientation in the neighborhood of a minutia is characterized by the local ridge orientation deviation at the location of the minutia and the average deviation in the neighborhood, i.e., $\left(d_{\theta}^{c}, \bar{d}_{\theta}\right)$. In this paper, we consider a circular neighborhood centered at the minutia whose radius is 64 pixels.

To study the distribution of minutiae neighboring to a central minutia, we represent the neighboring minutia in a local polar coordinate system as in [9] by its distance to the central minutia and the angle between the polar axis and the line connecting the neighboring minutiae and the central minutia, i.e., $(\rho, \phi)$.

It is worth mentioning that ideally, real minutiae and ridge orientation field in fingerprints should be used in analyzing and modeling the minutiae occurrence. In forensics, minutiae in latent fingerprints are manually marked by latent examiners. However, different examiners may not always mark the same minutiae in a fingerprint [18]. Moreover, it is expensive, tedious, and error-prone to manually mark minutiae and ridge orientation field in a large number of rolled or slap fingerprints. On the other hand, state of the art automated matchers, based on NIST's evaluations [4], perform almost as well as human experts in matching good quality fingerprints. This suggests that features extracted by the automated matchers are robust and reliable for good quality rolled fingerprints. In this paper, we thus choose good quality fingerprints, and automatically extract ridge orientation field and minutiae in these fingerprints to train the minutiae models. Note that the following analysis and the proposed model are also applicable if features from other sources are available (e.g., manually marked features, or features extracted by other automated methods).

\subsection{Occurrence Probability of Individual Minutiae}

We study the minutiae occurrence for each of the five major fingerprint classes separately by constructing the histograms of minutiae with respect to the local ridge deviation. To this end, we discretize the local ridge orientation deviation into 201 uniform bins between 0 and 1 with an interval of 0.005 , and set up two accumulators for each fingerprint class. Both accumulators consist of the 201 bins 


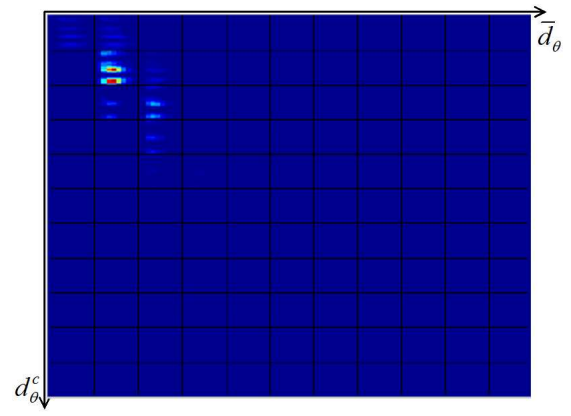

(a)

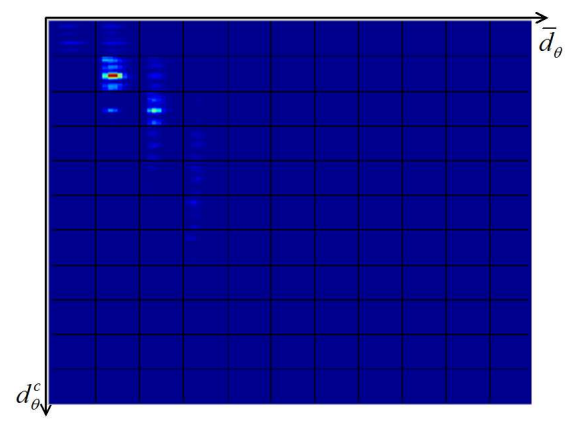

(d)

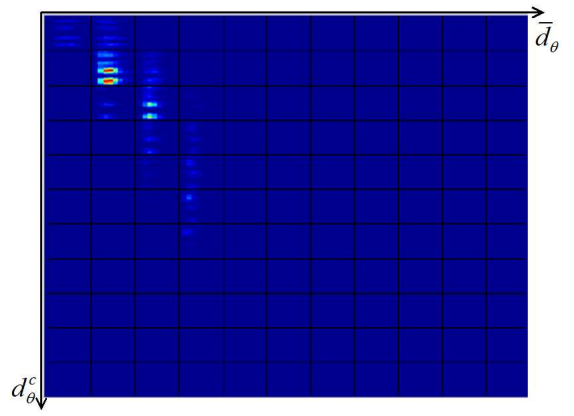

(b)

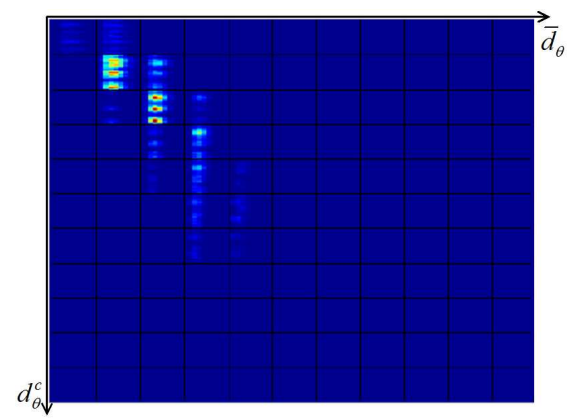

(e)

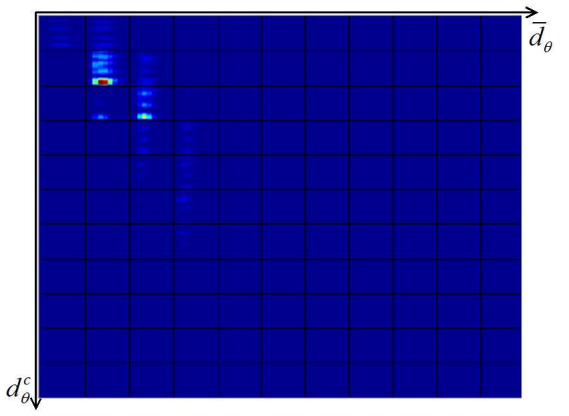

(c)

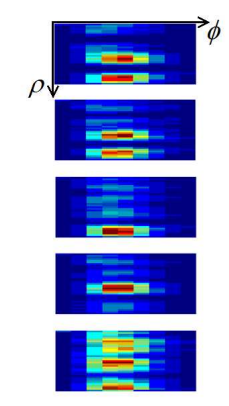

(f)

Figure 4. Occurrence probability maps of the nearest neighboring minutiae in fingerprints of type (a) arch, (b) tented arch, (c) left loop, (d) right loop, and (e) whorl computed from the fingerprints in NIST SD14 [1]. Each block corresponds to a type of neighborhood region defined by $\left(d_{\theta}^{c}, \bar{d}_{\theta}\right)$. The histogram in each block gives the occurrence probability of the nearest neighboring minutiae (in terms of their positions in the local polar coordinate system) in the corresponding type of neighborhood region. (f) distribution of the nearest neighboring minutiae in the neighborhood $\left(d_{\theta}^{c}=0.1, \bar{d}_{\theta}=0.1\right)$ for arch, tented arch, left loop, right loop, and whorl fingerprints (from top to bottom).

of local ridge orientation deviation. The first accumulator counts the local blocks according to the local ridge orientation deviation in the blocks, and the second accumulator counts the minutiae according to the local ridge orientation deviation at the locations of the minutiae. The occurrence probability map of individual minutiae with respect to the local ridge orientation deviation is obtained by taking the bin-to-bin quotient between the second and first accumulators. Each bin in the resulting probability map denotes the probability of a minutia appearing in a block which has the corresponding local ridge orientation deviation. Figure 3 shows the occurrence probability map of individual minutiae. We can observe that (i) minutiae appear in the blocks of different local ridge orientation deviations with different probabilities, and (ii) minutiae occurrence probabilities vary between different fingerprint classes.

\subsection{Distribution of Neighboring Minutiae}

In this paper, we assume that the distribution of neighboring minutiae also depends on the ridge orientation field in the neighborhood. To analyze this dependency, we characterize the ridge orientation field in the minutiae neighborhood by using two attributes $\left(d_{\theta}^{c}, \bar{d}_{\theta}\right)$, and discretize both of these attributes into 11 bins, i.e., from 0 to 1 with an interval of 0.1 . As a result, there are 121 $(11 \times 11)$ different types of neighborhood regions. For each region, we construct a histogram of the nearest neighboring minutiae in terms of their positions in the local polar coordinate system. Specifically, we divide the radius $(\rho)$ into 9 uniform bins (i.e., from 0 to 64 with an interval of 8 pixels, where 64 is the neighborhood radius) ${ }^{3}$, and the polar angle $(\phi)$ into 37 uniform bins (i.e., from 0 to 359 degrees with an interval of 10 degrees). Note that the bin $(\rho, \phi)=(0,0)$ indicates that there is no minutia in the neighborhood of the central minutia (in this paper, the radius of the circular neighborhood is 64 pixels).

Again, the neighboring minutiae distribution is investigated for each fingerprint class separately. The histograms of different types of neighborhood regions are computed for each fingerprint class based on the available training set of fingerprints of that class: given a minutia in a training fingerprint, (i) the type of its neighborhood is first determined according to the local ridge orientation field, (ii) its

\footnotetext{
${ }^{3}$ Note that we use an interval of 8 pixels (i.e., the local block size). In this way, we reduce the number of parameters to be estimated, but at the cost of lower resolution. This is a compromise between high accuracy and the limited number of available training samples.
} 
nearest minutia is then located and represented in the form of $(\rho, \phi)$, and finally, (iii) it is assigned to the corresponding nearest neighboring minutiae histogram bin of the corresponding type of neighborhood. If no minutia is found in its neighborhood, the bin $(\rho, \phi)=(0,0)$ is increased by one. Figure 4 shows the resulting occurrence probability maps of the nearest neighboring minutiae for five types of fingerprints (arch, tented arch, left loop, right loop, and whorl).

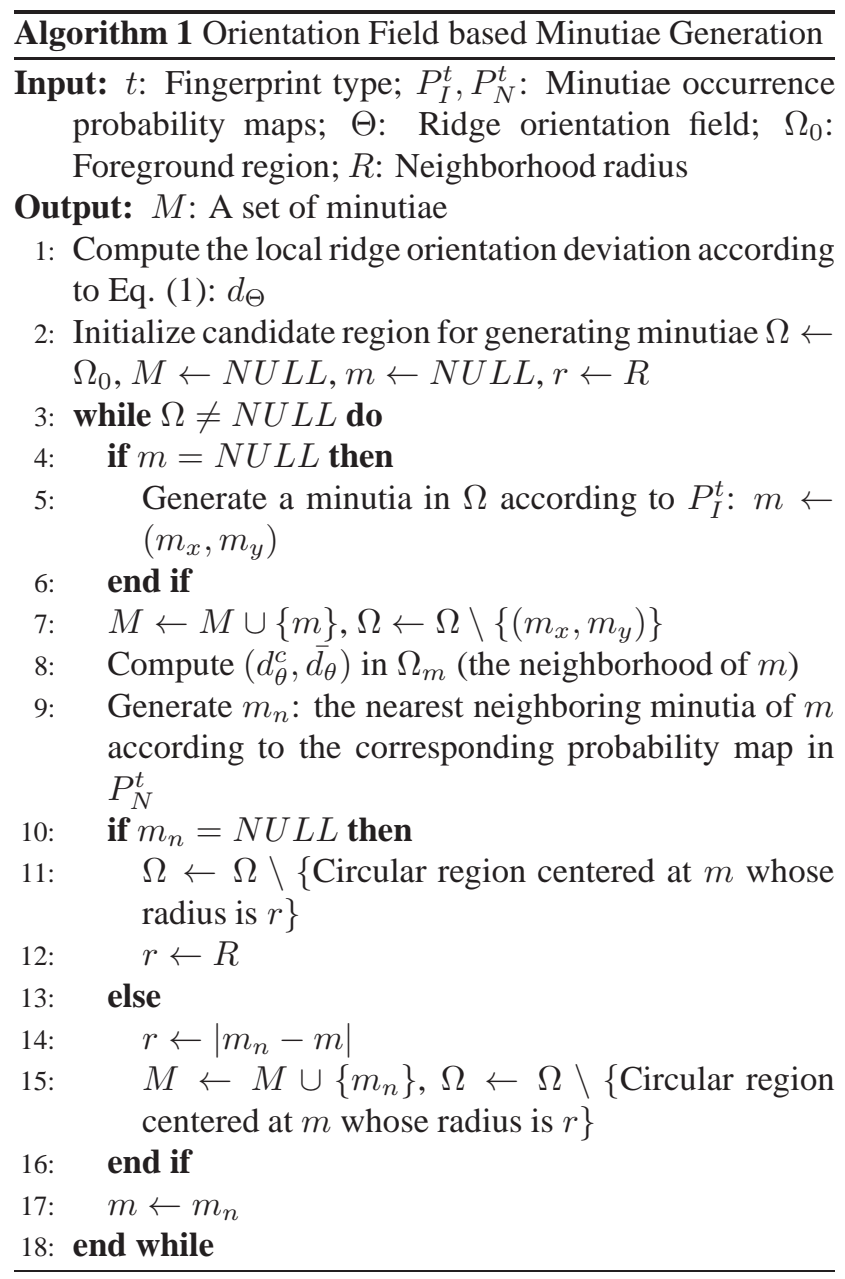

\section{Proposed Generative Model}

In the previous section, we constructed the occurrence probability maps for individual minutiae and nearest neighboring minutiae. Let us denote them as $P_{I}^{t}$ and $P_{N}^{t}$, respectively. Here $t \in\{A, T, L, R, W\}$ corresponding to arch, tented arch, left loop, right loop, and whorl fingerprints. In this section, we present the proposed generative model for minutiae, which randomly samples a set of minutiae from a given ridge orientation field based on the minutiae occurrence probability maps.

Given a block-wise ridge orientation field of a particular type of fingerprint (i.e., one of the five major fingerprint classes), the corresponding image area is initialized with an empty set of minutiae, and the local ridge orientation deviation in each block is computed. Then, the first minutia of the fingerprint is randomly generated according to $P_{I}^{t}$, and its nearest neighboring minutia is randomly generated according to $P_{N}^{t}$. More minutiae are gradually added by generating the nearest neighboring minutia of the previous minutia or generating a new individual minutia (if no neighboring minutia is generated for the previous minutia). This minutiae sampling process is iterated until the entire fingerprint image area has been considered. Algorithm 1 summarizes the process of sampling minutiae based on the proposed model, and Fig. 5 shows an example ${ }^{4}$.

\section{Experiments}

In this section, we compare the proposed minutiae model with the uniform model in [11], the pattern specific Gaussian mixtures based model in [7], and the SFinGe method [5]. The minutiae generated by these models and SFinGe are compared with the minutiae in the fingerprints in the NIST SD4 database [3] in terms of spatial statistics [12]. Specifically, we chose only the fingerprint images in the first session and whose NFIQ values are between 1 and 3 . The empirical statistics are computed based on the minutiae extracted by VeriFinger SDK 6.5 from these fingerprints (the minutiae outside the manually marked foreground regions are discarded). Minutiae are also randomly sampled by using the generative models for the same number of fingerprints, based on which the statistics of the generative models are calculated. The ridge orientation field required by the proposed model is the ridge orientation field of the chosen fingerprints in NIST SD4 extracted by using the method introduced in Section 3. As for SFinGe, images of 1,000 fingerprints including all the five major types are generated, based on which the minutiae spatial statistics are computed. Note that since SFinGe itself does not provide the minutiae locations in the synthetic fingerprints, we apply VeriFinger to extract the minutiae from the synthetic fingerprint images ${ }^{5}$.

Figure 6 shows the minutiae generated by the three different models for a given ridge orientation field of a whorl fingerprint. To quantitatively evaluate the models, fingerprints are divided into non-overlapping blocks, and the number of minutiae in each block is counted. The histogram of blocks is computed in terms of the number of minutiae in the blocks. The distance between the histogram

\footnotetext{
${ }^{4}$ The direction of minutiae in the proposed model can be easily determined according to the ridge orientation field and the minutiae type. The direction of ridge bifurcation is equal to the local ridge orientation at its location, while the direction of ridge ending is opposite to the local ridge orientation at its location. In this paper, we focus on the spatial distribution of minutiae, i.e., we consider only the minutiae locations.

${ }^{5}$ Master fingerprint images are used because they are free from noise. See [5] for more details of master fingerprint images generated by SFinGe.
} 


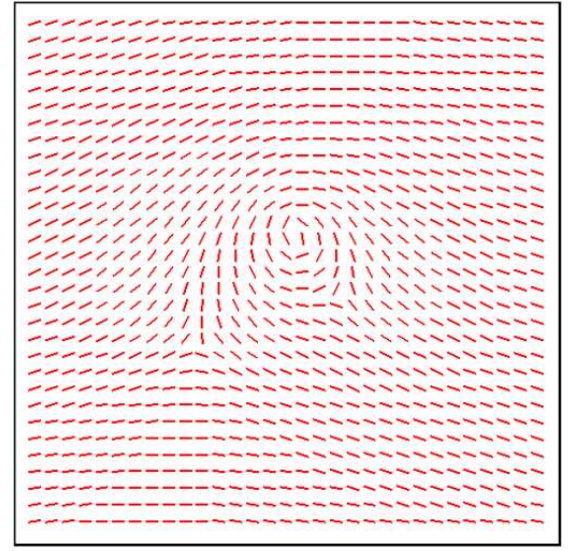

(a)

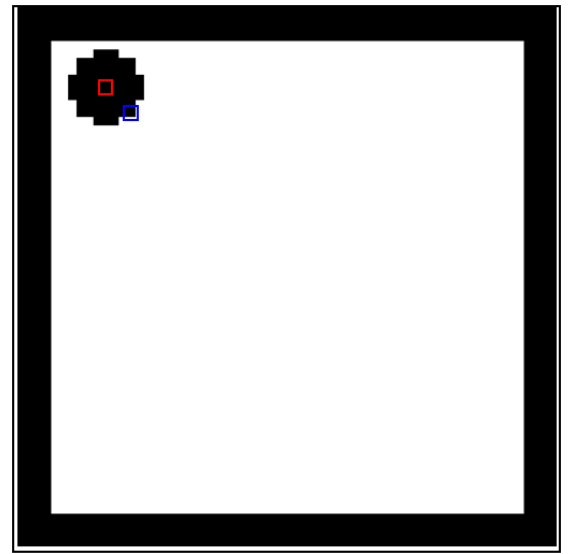

(d)

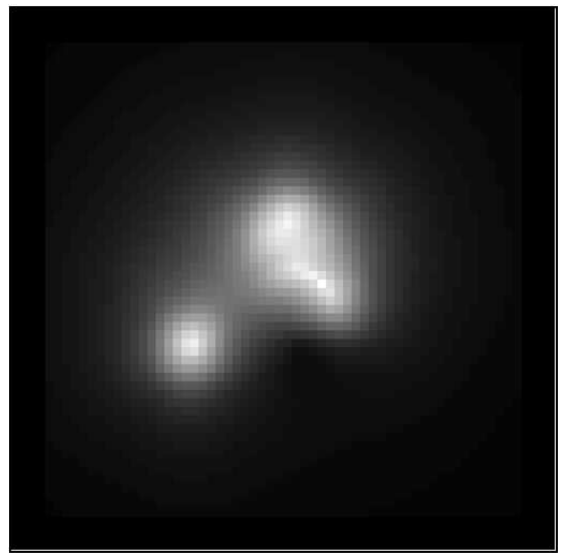

(b)

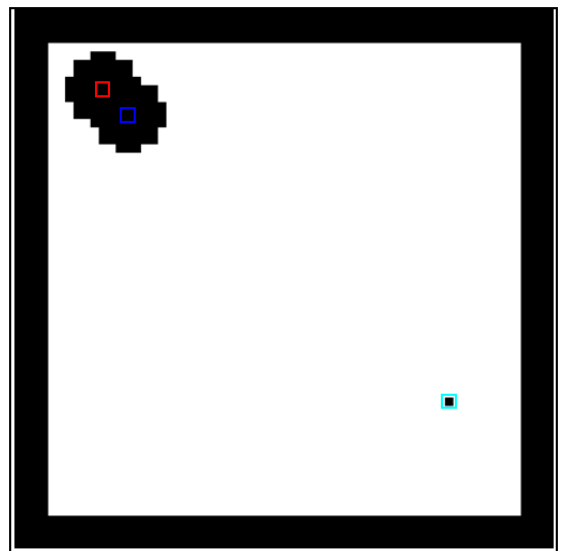

(e)

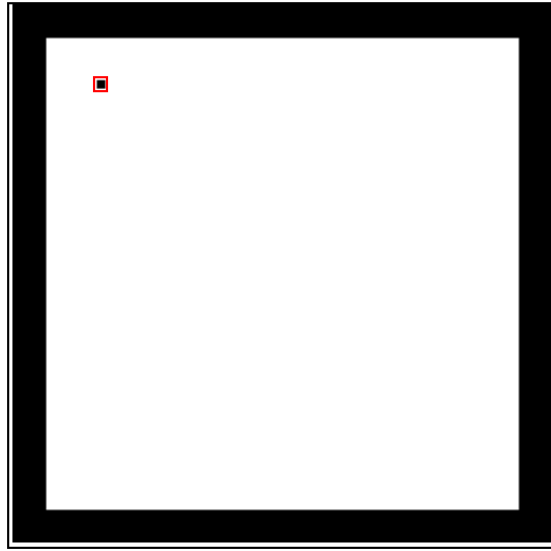

(c)

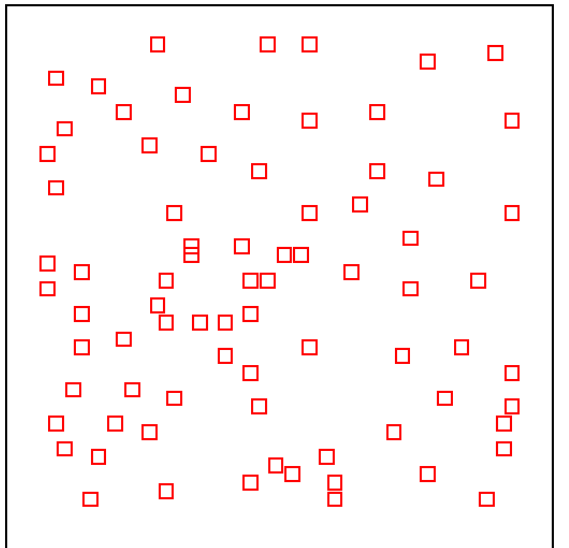

(f)

Figure 5. Sampling minutiae by using the proposed generative model. (a) a given ridge orientation field. (b) the corresponding local ridge orientation deviation map. (c)-(e) generation of the first, second, and third minutiae. The black regions denote the regions already considered, so no new minutiae can be added in them. (f) the final set of generated minutiae. Note that while the results in (b)-(e) are block-based (block size is $8 \times 8$ pixels), they are resized to the original image size for display purpose.

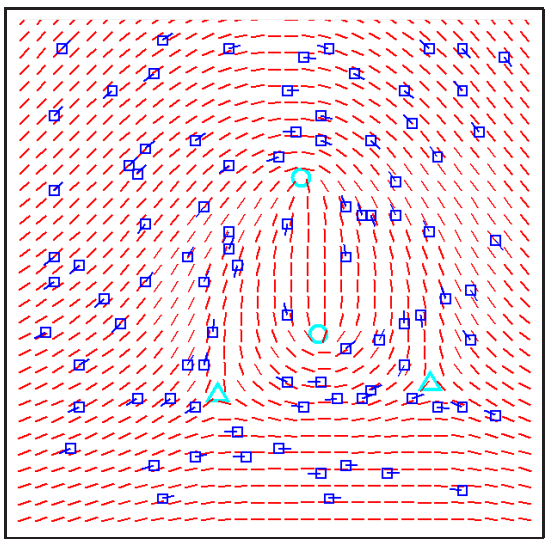

(a)

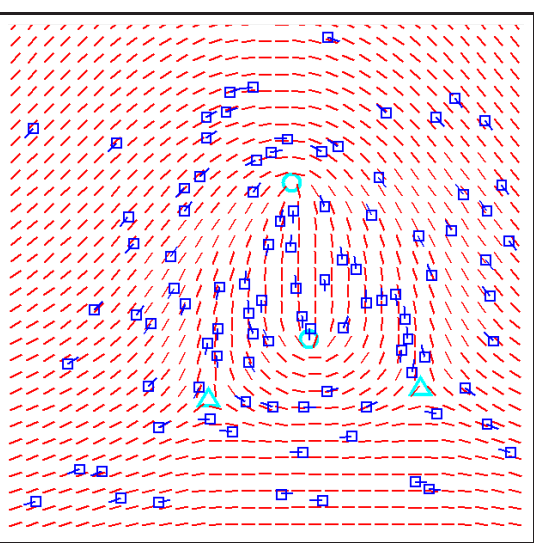

(b)

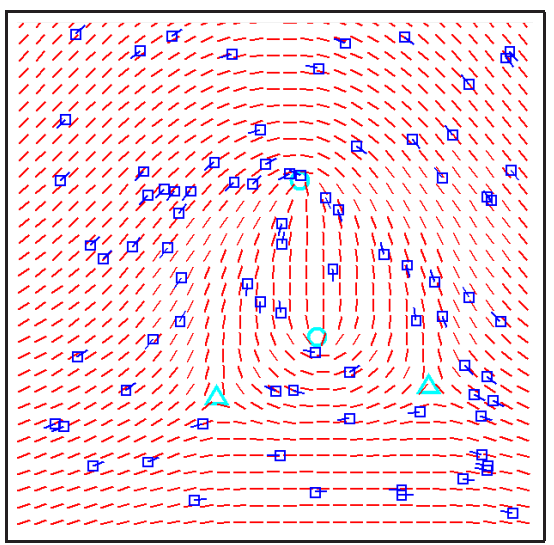

(c)

Figure 6. Given a ridge orientation field of a whorl fingerprint, the minutiae generated by (a) the proposed model, (b) the Gaussian mixtures based model [7], and (c) the uniform model [11]. 


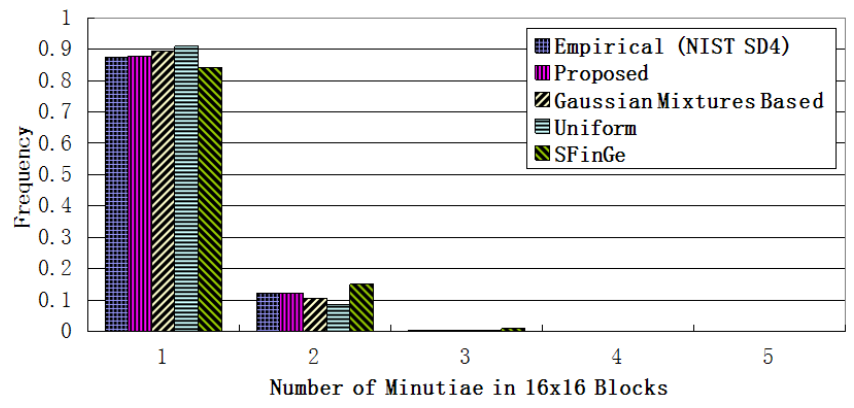

(a)

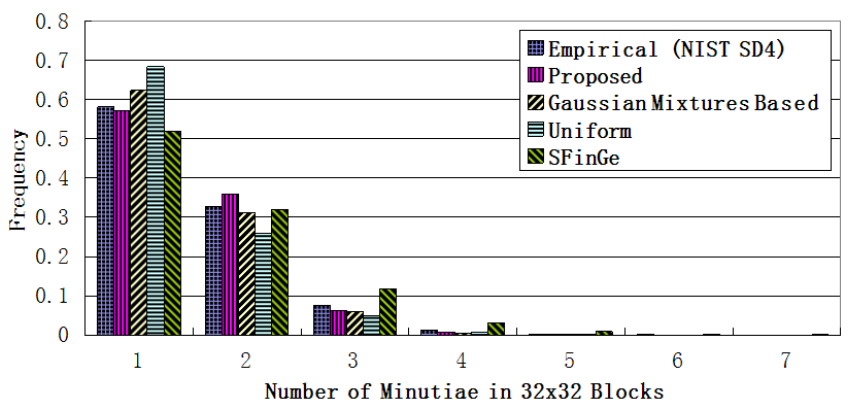

(b)

Figure 7. Comparison of the proposed model with the uniform model [11], the Gaussian mixtures based model [7], and SFinGe [5]. (a) and (b), respectively, show the histograms of $16 \times 16$ and $32 \times 32$ non-overlapping local blocks in terms of the number of minutiae appearing in the blocks.

\begin{tabular}{|c||c|c|c|c|}
\hline \multicolumn{1}{|c||}{ Block } & \multicolumn{4}{c|}{ Chi-Square Distance } \\
\cline { 2 - 5 } Size & Proposed & GM Based & Uniform & SFinGe \\
\hline $16 \times 16$ & 0.0005 & 0.0014 & 0.0032 & 0.0028 \\
$32 \times 32$ & 0.0028 & 0.0043 & 0.0123 & 0.0131 \\
\hline
\end{tabular}

Table 1. Chi-square distances between the empirical histograms (based on the fingerprints in NIST SD4) and the histograms obtained by the proposed model, the Gaussian mixtures (GM) based model [7], the uniform model [11], and SFinGe [5].

obtained from the minutiae generated by a model and the empirical histogram computed from the minutiae in the fingerprints in NIST SD4 indicates how well the model describes the minutiae distribution extracted from fingerprints. In our experiments, we compare different minutiae models based on the Chi-square distances between the empirical histogram and the histograms computed from the minutiae generated by these models.

Figure 7 shows the empirical histograms and the histograms obtained by different models and SFinGe. As can be seen, the histograms generated by the proposed model are more similar to the empirical ones. The Chi-square distances between these histograms are given in Table 1. The proposed model achieves the smallest distances among the three models and SFinGe. This suggests that the proposed model better captures the minutiae distribution extracted from fingerprints.

\section{Conclusions}

While minutiae are routinely used in fingerprint matching, understanding their spatial distribution is still an open problem. Previous studies on this topic have some deficiency in that they did not consider the correlation between minutiae (as a type of level-2 feature) and the ridge orientation field (as a type of level-1 feature). In this paper, we have analyzed the correlation between minutiae occur- rence and ridge orientation field, and explicitly incorporated such correlation into the minutiae distribution modelling. We proposed a generative model for minutiae. We have compared the proposed model with two typical models previously proposed in the literature; our experimental results show that the proposed model generates minutiae whose spatial distribution better resembles the minutiae distribution extracted from fingerprints.

We are currently improving the proposed model along three directions. (i) Using a larger training set and evaluating the stability of the model with respect to the training data size. The number of training fingerprints used in this paper is still relatively small, especially for arch and tented arch fingerprints. (ii) Applying the proposed model to other sources of fingerprint data, including both manually marked fingerprint features and the features extracted by additional automated matchers. Currently, the training and testing minutiae are both extracted by one single matcher, VeriFinger. Moreover, the minutiae extracted in singularity area (i.e., the area surrounding cores and deltas) often have low reliability. Researchers have typically avoided using the singularity area in studying the statistics of minutiae even when the minutiae are manually marked [16]. Therefore, we will also assess the impact of singularity area on the trained minutiae models. (iii) Using the minutiae density in local neighborhood to guide the generation of minutiae. In this paper, we consider only the first nearest minutiae. Considering more neighboring minutiae, we believe, will further improve the model fit.

\section{Acknowledgments}

This work is supported by the NIST 2012 Measurement Science and Engineering Research Grants Program and by a grant from the National Natural Science Foundation of China (No. 61202161). The authors thank all the anonymous reviewers for their helpful comments, and 
specially thank Austin Hicklin, Noblis for his constructive review and suggestions. The authors are also grateful to Dr. Jianjiang Feng and Soweon Yoon for their help in improving the paper, and to Shuiwang $\mathrm{Li}$, Xiutao $\mathrm{Li}$, and Yanyan Guo for their assistance in conducting some of the experiments.

\section{References}

[1] NIST Special Database 14, NIST Mated Fingerprint Card Pairs 2 (MFCP2). http://www.nist.gov/srd/ nistsd14.htm.

[2] Neurotechnology Inc., VeriFinger. http://www. neurotechnology.com.

[3] NIST Special Database 4, NIST 8-Bit Gray Scale Images of Fingerprint Image Groups (FIGS). http://www. nist. gov/srd/nistsd4.htm.

[4] Fingerprint Vendor Technology Evaluation (FpVTE) 2003. http://www.nist.gov/itl/iad/ig/fpvte03. Cfm.

[5] R. Cappelli, D. Maio, and D. Maltoni. Synthetic fingerprint database generation. In Proc. 16th International Conference on Pattern Recognition (ICPR'02), pages 1-4, 2002.

[6] R. Cappelli and D. Maltoni. On the spatial distribution of fingerprint singularities. IEEE Transactions on Pattern Analysis and Machine Intelligence, 31(4):742-448, 2009.

[7] Y. Chen and A. K. Jain. Beyond minutiae: A fingerprint individuality model with pattern, ridge and pore features. In Proc. 3rd International Conference on Biometrics (ICB'09), pages 523-533, June 2009.

[8] L. Hong, Y. Wan, and A. K. Jain. Fingerprint Image Enhancement: Algorithm and Performance Evaluation. IEEE Trans. Pattern Analysis and Machine Intelligence, 20(8):777-789, 1998.

[9] R. L. V. Hsu and B. Martin. An analysis of minutiae neighborhood probabilities. In Proc. IEEE 1st International Conference on Biometrics: Theory, Applications and Systems (BTAS'08), pages 1-6, 2008.

[10] D. Maltoni, D. Maio, A. K. Jain, and S. Prabhakar. Handbook of Fingerprint Recognition ( $2^{\text {nd }}$ Edition). Springer-Verlag, 2009.

[11] S. Pankanti, S. Prabhakar, and A. K. Jain. On the individuality of fingerprints. IEEE Transactions on Pattern Analysis and Machine Intelligence, 24(8):1010-1025, 2002.

[12] B. D. Ripley. Spatial Statistics. Wiley-Interscience, 2004.

[13] A. R. Roddy and J. D. Stosz. Fingerprint features - statistical analysis and system performance estimates. Proc. IEEE, 85(9):1390-1421, 1997.

[14] B. G. Sherlock and D. M. Monro. A model for interpreting fingerprint topology. Pattern Recognition, 26(7):1047-1055, 1993.

[15] D. A. Stoney. Measurement of Fingerprint Individuality. In H. C. Lee and R. E. Gaensslen, editors, Advances in Fingerprint Technology ( $2^{\text {nd }}$ Edition), pages 327-388. CRC Press, 2001.

[16] D. A. Stoney and J. I. Thornton. A Systematic Study of Epidermal Ridge Minutiae. Journal of Forensic Sciences, 32(5):1182-1203, 1987.
[17] E. Tabassi, C. Wilson, and C. Watson. Fingerprint Image Quality. NISTIR 7151, August 2004. http:// fingerprint.nist.gov/NFIS/ir_7151.pdf.

[18] B. T. Ulery, R. A. Hicklin, J. Buscaglia, and M. A. Roberts. Accuracy and Reliability of Forensic Latent Fingerprint Decisions. Proceedings of the National Academy of Sciences, USA, 108(19):77337738, 2011.

[19] Y. Wang and J. Hu. Global ridge orientation modeling for partial fingerprint identification. IEEE Transactions on Pattern Analysis and Machine Intelligence, 33(1):72-87, 2011.

[20] Y. Wang, J. Hu, and D. Phillips. A fingerprint orientation model based on 2d Fourier expansion (FOMFE) and its application to singular-point detection and fingerprint indexing. IEEE Transactions on Pattern Analysis and Machine Intelligence, 29(4):573-585, 2007.

[21] Q. Zhao and A. K. Jain. Model based separation of overlapping latent fingerprints. IEEE Transactions on Information Forensics and Security, 7(3):904-918, 2012.

[22] Q. Zhao, A. K. Jain, N. G. Paulter, and M. Taylor. Fingerprint image synthesis based on statistical feature models. In Proc. IEEE 5th International Conference on Biometrics: Theory, Applications and Systems (BTAS'12), pages 23-30, 2012.

[23] J. Zhou and J. Gu. A model-based method for the computation of fingerprints orientation field. IEEE Transactions on Image Processing, 13(6):821-835, 2004.

[24] Y. Zhu, S. C. Dass, and A. K. Jain. Statistical models for assessing the individuality of fingerprints. IEEE Transactions on Information Forensics and Security, 2(3):391-401, 2007. 